\title{
MANEJO LAPAROSCÓPICO DE MIOMATOSIS UTERINA EN PACIENTE CON SÍNDROME DE ROKITANSKY. REPORTE DE UN CASO Y REVISIÓN DE LA LITERATURA
}

\section{Laparoscopic management of uterine myomatosis in a patient with Rokitansky syndrome. Case report and review of the literature}

Guillermo Páez-López, $M D^{1}$; José Fernando De los Ríos-Posada, $M D^{2}$; Adriana María Arango-Martínez, $M D^{3}$; Juan David Castañeda-Roldán, $M D^{3}$; Eduardo Serna-Agudelo, $M D^{3}$; Ricardo Vásquez-Ruiz, MD $^{3}$; Luis Alberto Almanza-Payares, $\mathrm{MD}^{3}$; Gustavo Adolfo Calle-Gómez, $\mathrm{MD}^{3}$

Recibido: febrero 4/13 - Aceptado: noviembre 14/13

\section{RESUMEN}

Introducción: el síndrome de Mayer-RokitanskyKüster-Hauser (SMRKH) es un espectro de anomalías de los conductos müllerianos caracterizado por la ausencia congénita de útero y vagina en mujeres fenotípicamente normales, con cariotipo $46 \mathrm{XX}$, con un estado endocrino normal. Muchas de estas mujeres poseen remanentes uterinos. La presencia de miomas en dichos remanentes es un hallazgo de muy escasa ocurrencia por lo que se reporta el caso. Objetivo: describir la técnica quirúrgica laparoscópica y revisar el diagnóstico clínico y el diagnóstico diferencial.

Materiales y métodos: se presenta el caso de una mujer con diagnóstico previo de síndrome de

1 Fellow en cirugía endoscópica ginecológica, Universidad CES. Medellín, Colombia.

2 Ginecólogo endoscopista, Unidad Endoscopia Ginecológica, Clínica del Prado, Medellín, Colombia. Profesor Endoscopia Ginecológica, Universidad CES. Medellín, Colombia. cristidelos@une.net.co

3 Ginecólogo endoscopista, Unidad Endoscopia Ginecológica, Clínica del Prado, Medellín, Colombia. Profesor Endoscopia Ginecológica, Universidad CES. Medellín, Colombia.
Mayer-Rokitansky-Küster-Hauser (SMRKH), quien consulta a la Unidad de Endoscopia Ginecológica de la Clínica del Prado en Medellín, institución privada de tercer nivel de complejidad y que es centro de referencia para manejo laparoscópico de pacientes con dolor pélvico crónico del departamento de Antioquia, Colombia. La ecografía reporta una masa sólida de ocho centímetros en la pelvis. Durante la laparoscopia se encuentra un mioma en uno de los remanentes uterinos. Se realiza miomectomía y se resecan ambos remanentes por laparoscopia. Se realizó una búsqueda sistemática de la literatura a través de Medline en la página de Pubmed utilizando los siguientes términos: "Mayer-Rokitansky- Hauser Syndrome", "leiomyoma" y "laparoscopy".

Resultados: se encontraron 25 artículos en los últimos 20 años de los cuales 17 estaban relacionados con el tema de manera directa; de estos, 2 incluían el manejo por laparoscopia.

Conclusiones: la presencia de leiomioma en un remanente uterino de una paciente con MRKH es poco frecuente. El caso aquí presentado es el segundo reportado en Colombia y el primero manejado 
por vía laparoscópica. En pacientes con síndrome de Mayer-Rokitansky-Küster-Hauser y masas pélvicas, la laparoscopia es una opción diagnóstica y terapéutica por considerar.

Palabras clave: síndrome Mayer-Rokitansky-Küster-Hauser-Rokitansky, leiomioma, laparoscopia.

\section{ABSTRACT}

Introduction: The Mayer-Rokitansky-KüsterHauser (MRKH) syndrome is a spectrum of müllerian duct anomalies characterized by the congenital absence of the uterus and vagina in phenotypically normal women with a $46 \mathrm{XX}$ karyotype and normal endocrine status. Many of these women have uterine remnants. The presence of fibroids in those remnants is a very rare finding, hence this case report.

Objective: To describe the laparoscopic surgical technique and review the clinical and differential diagnoses.

Materials and methods: Female patient with a prior diagnosis of MRKH syndrome who presented to the Gynecological Endoscopy Unit at Clinica del Prado, a private, level 3 institution in Medellin, which is a referral center for the laparoscopic management of patients with chronic pelvic pain in the Department of Antioquia, Colombia. A solid, nine-centimeter pelvic mass was found on ultrasound. During laparoscopy, a leiomyoma was found in one of the uterine remnants. Laparoscopic myomectomy was performed and the two remnants were resected. A systematic review of the literature was conducted through Medline in the Pubmed site using the following terms: "Mayer-RokitanskyHauser Syndrome", "leiomyoma" and laparoscopy.

Results: In total, 25 articles of the past 20 years were found; of those, 17 were related directly to the topic, and 2 of these included laparoscopic management.

Conclusions: The presence of leiomyoma in a uterine remnant in a patient with MRKH syndrome is infrequent. The case presented here is the second reported in Colombia and the first managed laparoscopically. In patients with the Mayer-Rokitansky-Küster-Hauser syndrome and pelvic masses, laparoscopy is a diagnostic and therapeutic option.

Key words: Mayer-Rokitansky-Küster-Hauser, leiomyoma, laparoscopy.

\section{INTRODUCCIÓN}

El síndrome de Mayer-Rokitansky-Küster-Hauser (SMRKH) es un espectro de anomalías de los conductos müllerianos caracterizado por la ausencia congénita de útero y vagina, en mujeres fenotípicamente normales, con cariotipo $46 \mathrm{XX}$, y un estado endocrino normal dado que los ovarios están usualmente presentes y funcionales. Aunque su etiología es desconocida parece existir una activación de la hormona antimülleriana o sus receptores $(1,2)$. Se presenta en una de cada 4.000-5.000 mujeres nacidas vivas y en la actualidad se reconocen dos variantes del síndrome: el tipo I que se caracteriza por una aplasia aislada de los dos tercios superiores de la vagina y el útero, y el tipo II, en el que además de dichos hallazgos se encuentran otras anomalías en el tracto urinario, anomalías esqueléticas y en particular vertebrales, defectos auditivos y, más raramente, anomalías cardiacas y digitales como sindactilia o polidactilia $(3,4)$.

En vez del útero se encuentran los remanentes müllerianos que están compuestos de tejido muscular semejante al miometrio uterino y hasta un 5\% de estas mujeres pueden tener pequeñas cantidades de endometrio activo, que se acompaña de dolor pélvico cíclico. El crecimiento de miomas en mujeres con el SMRKH sin remanentes uterinos es infrecuente; puede ocurrir por tener los niveles hormonales normales (5-8). Hay reportes en los que se describen miomas en el ligamento redondo u ocupando el fondo de saco posterior (9). Igualmente, hay reportes de mujeres posmenopáusicas con el SMRKH quienes reciben terapia de reemplazo hormonal y desarrollan miomas (10). En tales casos está indicado remover el tumor junto con los remanentes uterinos (11), puesto que la sola extirpación 
del tumor con la conservación del tejido muscular del rudimento uterino puede dar lugar a recidivas de la enfermedad (12).

Es importante conocer esta patología sobre todo en pacientes con amenorrea primaria ya que es causa de masa pélvica que requiere un diagnóstico diferencial con otras causas de masa abdominopélvica, como también conocer la posibilidad de manejo laparoscópico como alternativa al acceso quirúrgico por laparotomía.

La presentación de este caso tiene como objetivo la descripción de la técnica quirúrgica empleada, y hacer una búsqueda de la literatura respecto a las publicaciones relacionadas con el tema, el desempeño operativo de los métodos utilizados para su diagnóstico, y su diagnóstico diferencial.

\section{REPORTE DEL CASO}

Paciente de 32 años, quien consultó por amenorrea primaria y dolor pélvico de dos años de evolución a la Unidad de Endoscopia Ginecológica de la Clínica del Prado, institución privada de tercer nivel de complejidad ubicada en Medellín, Colombia, centro de referencia del departamento de Antioquia, la cual atiende pacientes afiliados al aseguramiento contributivo y particulares. Entre los antecedentes se reportaba un síndrome de MayerRokitansky-Küster-Hauser diagnosticado 20 años atrás mediante laparoscopia, la cual reportó ovarios normales, cuernos uterinos rudimentarios bilaterales, y agenesia del tercio superior de la vagina, sin gestaciones previas; como antecedentes familiares de importancia mencionó que dos tías maternas tenían el mismo síndrome.

Como hallazgo positivo en la valoración abdominal se encontró una masa pélvica de ocho centímetros, móvil, dura y levemente lateralizada a la derecha. En relación con la evaluación pélvica se encontraron unos genitales externos de aspecto normal y en el tacto vaginal se encontró una vagina de seis centímetros que terminaba en saco ciego y una masa de ocho centímetros ocupando el fondo de saco de Douglas, no dolorosa con la movilización.
La ecografía transvaginal reportó mioma de 89 × 79 $\mathrm{mm}$ en fondo de saco y ovarios de aspecto normal.

Con diagnóstico de miomatosis uterina y agenesia de tercio superior de vagina en una paciente con síndrome de MRKH, se programa para miomectomía y resección de cuernos uterinos rudimentarios mediante laparoscopia.

Se realiza incisión infraumbilical, se introduce aguja de veress y trocar de $10 \mathrm{~mm}$ con una presión de $15 \mathrm{~mm}$; se observa un mioma de ocho centímetros localizado en el rudimento uterino derecho, con trompa y ovario ipsilaterales de aspecto normal. En el lado izquierdo se observó un rudimento uterino, trompa y ovario normales (figura 1).

Se adicionaron dos puertos laterales izquierdos y un puerto lateral derecho, se realizó miomectomía y resección de ambos cuernos rudimentarios utilizando energía bipolar y tijeras (figura 2). A las tres horas del procedimiento la paciente fue dada de alta con manejo ambulatorio utilizando analgésicos y anti-inflamatorios. Una semana más tarde, en la revisión posoperatoria, fue hallada en buenas condiciones.

El reporte de patología informó remanente uterino con mioma.

Aspectos éticos: se solicitó autorización a la paciente y a la Clínica del Prado para publicar las fotos y la presentación del caso. Se garantizó la confidencialidad de la información para proteger los derechos de la paciente.

\section{MATERIALES Y MÉTODOS}

Con las palabras clave: "Mayer-Rokitansky-HauserSyndrome”, "leiomyoma” y "laparoscopy” se realizó una búsqueda sistemática de la literatura en inglés y español a través de las bases de datos Medline vía Pubmed, Embase, Lilacs que se relacionara con el síndrome de Rokitanski, leiomioma y cirugía, además de diagnóstico y diagnóstico diferencial.

\section{RESULTADOS}

Se encontraron 25 artículos en los últimos 20 años de los cuales 17 estaban relacionados con el tema 


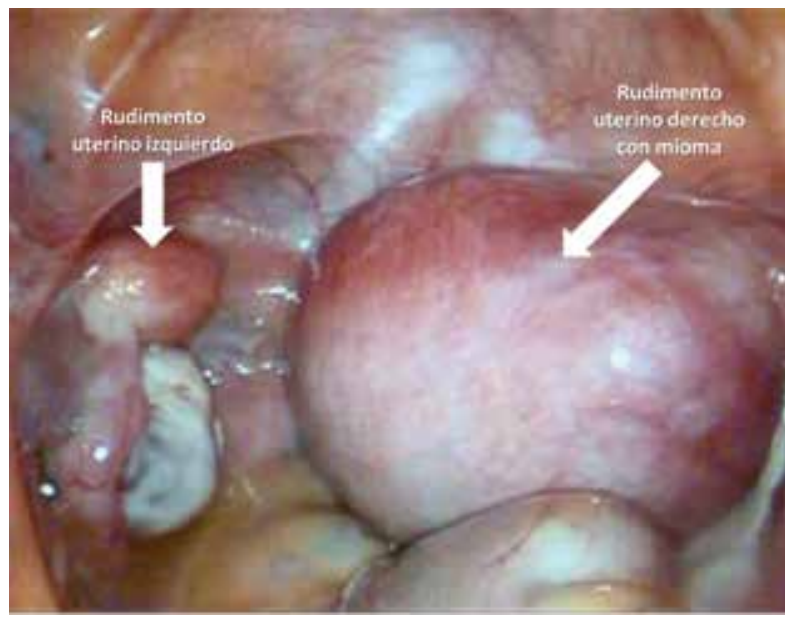

Figura 1. Mioma en cuerno uterino rudimentario en paciente con síndrome de Rokitansky

de manera directa; de estos, 2 incluían el manejo por laparoscopia $(11,13)$. Todos corresponden a reportes de casos y una sola revisión de tema.

\section{DISCUSIÓN}

El presente caso ilustra una paciente con SMRKH y leiomioma en el remanente mülleriano con síntomas de dolor pélvico y cuyo diagnóstico se sospechó por ecografía. La realización de la laparoscopia sirvió como diagnóstico y tratamiento permitiendo su manejo ambulatorio y buena evolución. Según la literatura revisada este reporte de caso es el primero en Colombia y el tercero en el mundo de una paciente con SMRKH y leiomioma realizado por laparoscopia $(13,14)$.

El síndrome de Mayer-Rokitansky-Küster-Hauser (SMRKH) fue descrito por Mayer, y estudios adicionales fueron presentados por Rokitansky, Küster y Hauser (Rokitansky 1838). Sin embargo, el primer caso de una paciente con miomatosis y síndrome de Rokitansky fue publicado por Beecham et al. en 1977 (15); reportes posteriores se presentaron con otros autores $(13,16,17)$. Desde entonces no existen más de 20 casos reportados en la literatura mundial (18-20). A nivel nacional, González et al. reportaron el primer caso de una paciente con el síndrome a quien se le diagnosticó un mioma en uno de los remanentes y le fue extirpado por laparotomía (20).

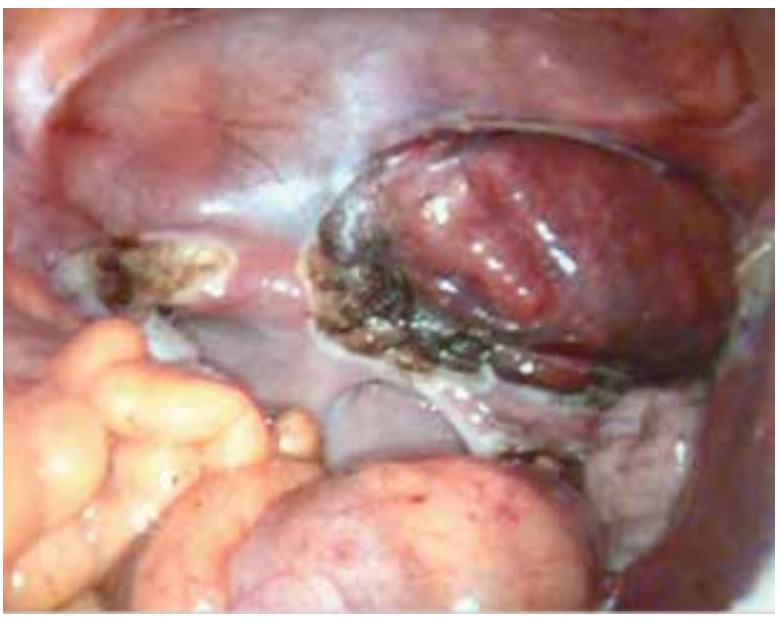

Figura 2. Visión final de la pelvis tras la resección de ambos rudimentos uterinos

Como se describió previamente, es poco frecuente encontrar leiomiomas en los remanentes uterinos, los cuales pueden ser asintomáticos o manifestarse como una masa de crecimiento progresivo y dolor pélvico agudo o crónico (21, 22), con riesgo de torsión del remanente mülleriano, especialmente cuando estos miomas son grandes y pedunculados. Los miomas tienen características anatómicas e histológicas idénticas a los encontrados en pacientes sin el síndrome (23).

Para el abordaje diagnóstico se ha informado el uso de la ecografía transvaginal o la resonancia magnética; sin embargo, no hay información sobre la sensibilidad y especificidad (24), pero la laparoscopia es la técnica ideal (estándar de oro) para diferenciar las masas anexiales de fibromas y otros tumores en pacientes con SMRKH, lo cual permitiría una opción diagnóstica y terapéutica previo consentimiento informado (11, 14, 15, 17, 25-27).

Dado que los ovarios son normales y hay adecuada función esteroidogénica, se deben descartar enfermedades ováricas en aquellas pacientes con SMRKH que presenten masas pélvicas, puesto que hay reportes de cáncer de ovario en pacientes con este síndrome (14, 28-31). El seguimiento también es importante para evitar complicaciones como la torsión o la degeneración del mioma (14). 


\section{CONCLUSIONES}

Tras una búsqueda exhaustiva en la literatura se encuentra que el caso aquí presentado es el segundo reportado en Colombia y el primero manejado por vía laparoscópica.

En pacientes con síndrome de Mayer-Rokitansky-Küster-Hauser el diagnóstico de leiomioma en un remanente uterino, aunque es poco frecuente, debe ser considerado si tiene historia de dolor y masa pélvica. La miomectomía y resección de los cuernos rudimentarios por laparoscopia es una alternativa a la laparotomía.

\section{REFERENCIAS}

1. Egarter C, Huber J. Successful stimulation and retrieval of oocytes in patient with Mayer-Rokitnsky-HauserKüster syndrome. Lancet. 1988;1:1283.

2. Ludwig KS. The Mayer-Rokitansky-Küster-syndrome. An analysis of its morphology and embryology. Part 1: Morphology. Arch Gynecol Obstet. 1998; 262:1-26.

3. Tarry WF, Duckett JW, Stephens FD. The MayerRokitansky syndrome: pathogenesis, classification and management. J Urol. 1986;136:648-52.

4. Ramírez JC, Puerta AJ, Rebollo A, Benítez R, Peña A, de la Macorra JC. Rokitanskys syndrome in association with reno-ureteral abnormalities. Teratogenic period. Eur Urol. 1987;13:346-50.

5. Powell B, Cunnane MF, Dunn Lk, Corson SL. Leiomyoma uteri in a rudimentary uterine horn in a woman with the Rokitansky-Küster-Hauser syndrome. A case report. J Reprod Med. 1988;33:493-4.

6. Malik E, Mangold MF, Rossmanith WG. Laparoscopic extirpation of an aplastic uterus in a patient with Mayer-Rokitansky-Küster-Hauser syndrome. Hum Reprod. 1997;12:1298-9.

7. Dandu S, Jones SE, Okeahialam MG. RokitanskyKüster-Hauser syndrome associated with chromosomal abnormality and fibroid arising from the rudimetary uterine horn. J Obstet Gynecol. 2000;20:98.

8. Lau J, Molina W, Andrino R, Reyes Martinez CA. Síndrome de Mayer-Rokitansky-Küster-Hauser. Revcog. 2009;14:11-4.
9. Rhee CS, Kim JS, Woo SK, Suh SJ. MRI of round ligament leiomyoma associated with MayerRokitansky-Küster-Hauser syndrome. Abdom Imaging. 1999;24:202-2044.

10. Edmonds DK. Multiple fibroids in a postmenopausal woman with Mayer Rokitansky-Küster-Hauser syndrome. J Pediatr Adolesc Gynecol. 2003;16:65-6.

11. Deligeoroglou E, Kontoravdis A, Makrakis E, Christopoulos P, Kountouris A, Creatsas G. Development of leiomyomas on the uterine remnants of two women with Mayer-Rokitansky-Küster-Hauser syndrome. Fertil Steril. 2004; 81:1385-7.

12. Galajdova L, Verberken K, Dhont M. Recurrent multiple leiomyomata in a patient with MayerRokitansky-Küster-Hauser syndrome. J Obstet Gynaecol. 2003;23:448-9.

13. Tsin D, Waters T, Granato R. Laparoscopic myomectomy in a patient with Mayer-RokitanskyKüster-Hauser Syndrome. J Am Assoc Gynecol Laparosc. 2000;7:411-3.

14. Lanowska M, Favero G, Schneider A, Kholer C. Laparoscopy for differential diagnosis of a pelvic mass in a patient with Mayer-Rokitansky-Küster-Hauser syndrome. Fertil Steril. 2009;91:e17-e18.

15. Beecham CT, Skiendzielewski J. Myoma in association with Mayer-Rokitansy-Küster syndrome. Am J Obstet Gynecol. 1977;129:346-8.

16. Yan CM, Mok KM. Uterine fibroids and adenomyosis in a woman with Rokitansky-Küster-Hauser syndrome. J Obstet Gynaecol. 2002;22:561-2.

17. Ito Y, Komori S, Horiuchi I, Kinuta T, Hori M, Wada C. Solid pelvic tumor in a woman with MayerRokitansky-Küster-Hauser syndrome. Arch Gynecol Obstet. 2006;274:252-4.

18. Papa G, Andreotti M, Giannubilo SR, Cesari R, Ceré I, Tranquilli AL. Case Report and surgical solution for a voluminous uterine liomyoma in a woman with complicated Mayer-Rokitansky-Küster-Hauser syndrome. Fertil Steril. 2008;90:2014-6.

19. Forsnes EV, Derrough KR, O'Donoghue M. A mitotically active myoma In Rokitansky-KüsterHauser syndrome. Obstet Gynecol. 1999;94:862. 
20. González MA, Pérez M. Leiomioma uterino en paciente con síndrome de Mayer-Rokitansky-KüsterHauser: Reporte de caso. Rev Colomb Obstet Ginecol. 2010;61:359-62.

21. Jadoul P, Pirard C, Squifflet J, Smets M, Donnez J. Pelvic mass in a woman with Mayer-Rokitansky-KüsterHauser syndrome. Fertil Steril 2004; 81:203-4.

22. Enatsu A, Harada T, Yoshida S, Iwabe T, Terakawa N. Adenomyosis in a patient with the Rokitanky-KüsterHauser syndrome. Fertil Steril 2000; 73:862-3.

23. Fletcher HM, Campbell-Simpson K, Walcott D, Harroit J. Mullerian Remnant in women with MayerRokitansky-Küster-Hauser syndrome. Obstet Gynecol 2012;119:483-5.

24. Morcel K, Camborieux L. Mayer-Rokitansky-KüsterHauser (MRKH) syndrome. Orphanet J Rare Dis. 2007;2:13.

25. Reinhold C, Hricak H, Forstner R, Ascher SM, Bret PM, Meyer WR. Primary amenorrhea: evaluation with MR Imaging. Radiology. 1997;203:383-90.
26. Rawat KS, Buxi TBS, Yadav A, Ghuman SS, Dhawan S. Large leiomyoma in a woman with MayerRokitansky-Küster-Hauser syndrome. J Radiol Case Rep. 2013;7:39-46.

27. Farber M, Stein A, Adashi E. Rokitansky-KüsterHauser syndrome and Leiomyoma uteri. Obstet Gynecol 1978;51:70-3.

28. Ghirardini G, Magnani A. Mayer-Rokitansky-KüsterHauser syndrome and ovarian cancer. Report of a case. Clin Exp Obstet Gynecol 1995;22:247-8.

29. Tsaur GT, Lee MH, Su SL,Wu MJ, Huang TW. MayerRokitansky-Küster-Hauser syndrome with immature teratoma of the ovary at age of 4 years. Gynecol Oncol 1995;56:456-9.

30. Küster H. Uterusbipartitussolidusrudimentarius cum vagina solida. Z Geburtshilfe Gynakol. 1910; 67:692-718.

31. Fukuda J, Kumazawa Y, Fujimoto T, Tanaka T. MayerRokitansky-Küster Hauser syndrome complicated by either uterine leiomyoma or ovarian tumor. J Obstet Gynaecol. 2010;36:191-4. 